\title{
A Importância das Relações Parentais Frente aos Problemas de Comportamento na Infância: Convergências Teóricas
}

\author{
Luiza Maria de Oliveira Braga Silveira"a \& Adriana Wagner ${ }^{b}$ \\ ${ }^{a}$ Fundação Universidade Federal de Ciências da Saúde de Porto Alegre, Porto Alegre, Brasil \& \\ ${ }^{\mathrm{b}}$ Universidade Federal do Rio Grande do Sul, Porto Alegre, Brasil
}

\begin{abstract}
RESUMO
Este artigo destaca a importância das relações parentais associadas aos problemas de comportamento na infância à luz de três modelos: $\mathrm{O}$ modelo da coerção, psicanalítico e ecológico-sistêmico. $\mathrm{O}$ primeiro destaca a importância das interações pais-filhos, de variáveis ambientais e suas interrelações. Já o modelo psicanalítico ressalta o desenvolvimento do ego e superego, da privação e convergência das pulsões agressivas e libidinais para o desenvolvimento normal. Por fim, o modelo ecológico-sistêmico destaca as características individuais, a história familiar parental e as relações conjugais e com a rede de apoio. Os modelos teóricos foram discutidos com vistas ao consenso acerca das relações parentais e à ampliação da compreensão desse fenômeno, destacando a importância de integrar aspectos psíquicos e ambientais, reiterando sua interdependência.
\end{abstract}

Palavras-chave: problemas de comportamento; infância; preditores.

\section{ABSTRACT \\ The Importance of Parent-Child Relationship to Child Behavior Problems: Theoretical Agreements}

This article highlights the importance of parent-child relationships associated to child problem behaviors through three theoretical models: coercion, psychoanalytical and ecological-systemic. The coercion model emphasizes the importance of parent-child interactions, the environmental variables and its interrelations. The psychoanalytical model emphasizes the significance of the ego and superego development, of the deprivation and of the convergence of aggressive and sexual drives to the normal development. Finally, the ecological-systemic model emphasizes individual characteristics, parent's family history and marital and support network relationships. The models were discussed in order to sort out a consensus about parent-child relationships and improve the comprehension of this phenomenon, highlighting the importance of psychological and environmental aspects, while reaffirming its interdependency.

Keywords: behavior problems; childhood; predictors.

Estudos e discussões sobre a importância da família na educação de crianças e adolescentes têm sido cada vez mais frequentes nos meios de comunicação. Normalmente, esses estudos buscam conhecer e destacar o valor das ações educativas dos pais e da família frente às dificuldades de comportamento dos filhos - seja na promoção de comportamentos adequados, seja na identificação de causas daqueles que não são considerados desejáveis. A maioria dos estudos que discutem esse tema da educação familiar centra-se nas estratégias educativas parentais disfuncionais; ou seja, nas práticas educativas parentais, associando-as ao rendimento escolar e aos transtornos de comportamento em adolescentes (Deslandes, Bouchard, \& St-Amant, 1998; Dodge, Petit, \& Bates, 1994; Ferreira \& Marturano, 2002; Glaskow, Dornbusch, Troyer, Steinberg, \& Ritter, 1997; Olson, Bates, Sandy, \& Lanthier, 2000; Petit, Bates, \& Dodge, 1997; Zdanowicz, Pascal, \& Reynaert, 2004).

\footnotetext{
* Endereço para correspondência: Luiza Maria de Oliveira Braga Silveira: luizasilveira@ @otmail.com
} 
O fracasso dos agentes socializadores (família e escola) na sua tarefa de socializar as crianças pode expressar-se através de quadros psicológicos diversos, dentre eles os problemas de comportamento na infância. Embora seja um tema bastante estudado, a literatura não oferece um conceito definido desse fenômeno, limitando-se, muitas vezes, aos instrumentos utilizados para medir tal construto (Alvarenga, \& Piccinini, 2001; Davidov \& Grusec, 2006; Ferreira \& Marturano, 2002). De forma geral, os problemas de comportamento são compreendidos como a dificuldade em estabelecer e manter relações interpessoais, podendo revelar-se como problemas de externalização (agressividade, hiperatividade, desobediência e comportamento delinquente) ou de internalização (retraimento, submissão, falta de iniciativa e expressão de afeto) (Achenbach, 1991; Achenbach \& Howell, 1993; Alvarenga \& Piccinini, 2001; Bolsoni-Silva \& Marturano, 2002; Ferreira \& Marturano, 2002). Os problemas de externalização são aqueles que consistem na transgressão de normas morais, realização de atos de violência e danos a terceiros; enquanto os problemas de internalização referem-se a condutas de isolamento e rechaço social, insegurança e exclusão, como não se relacionar com outros, ou ser constantemente enganado ou injustiçado (Ceballos \& Rodrigo, 2003; Hoffman, 1994). Muitas vezes, os primeiros são percebidos com maior frequência visto que perturbam e repercutem mais no ambiente social imediato (colegas, amigos, professores, familiares e pessoas em geral).

Estudos têm revelado que os problemas de comportamento são, frequentemente, associados a dificuldades na escola e na vida familiar de crianças e adolescentes, tais como: fracasso escolar (Deslandes e cols., 1998; Ferreira \& Marturano, 2002; Glaskow e cols., 1997), problemas de relacionamentos interpessoais (Bolsoni-Silva \& Marturano, 2002; Deslandes e cols., 1998; Dodge e cols., 1994; Grusec \& Goodnow, 1994) e violência intra e extrafamiliar (Belsky, 1990; Belsky, Jaffee, Hsieh, \& Silva, 2001; Patterson, Reid, \& Dishion, 1992; Petit e cols., 1997).

Entende-se que os problemas de comportamento implicam no aumento de conflitos, assim como na dificuldade da criança em manejá-los, e por isso revelam dificuldades na socialização (Bordin, Mari, \& Caeiro, 1995). Por isso, é de grande importância a busca pelo conhecimento dos processos que podem gerar tais dificuldades comportamentais e assim intervir e favorecer a qualidade das interações familiares e entre os sistemas que se ligam à família no processo de socialização das crianças. Apesar de, tanto os pro- blemas de externalização como de internalização associarem-se às dificuldades apontadas, os modelos teóricos apresentados a seguir tendem a centrar-se nos quadros referentes à externalização. Dessa forma, este artigo pretende destacar, a partir da etiologia dos problemas de comportamento, a importância das relações parentais frente aos mesmos, especialmente na infância, baseando-se em três modelos explicativos: da coerção, psicanalítico e ecológico-sistêmico. As perspectivas teóricas foram escolhidas de acordo com a existência de estudos e pressupostos teóricos que embasem e se associem ao quadro em questão.

\section{Os problemas de comportamento segundo 0 modelo da coerção e a abordagem dos sistemas dinâmicos}

Um modelo bastante utilizado em estudos nacionais e internacionais para a compreensão dos problemas de comportamento é o de Patterson, Reid \& Dishion (1992) (Granic \& Patterson, 2006; Pacheco, 2002; Pacheco, Alvarenga, Reppold, Piccinini, \& Hutz, 2005), chamado de "modelo da coerção". Segundo este modelo, tanto os comportamentos prósociais como antissociais são aprendidos nas interações familiares. Estas interações são favorecidas pelo ambiente, que neste caso contempla o contexto familiar, as características desenvolvimentais do próprio indivíduo e dos familiares, as relações conjugais e também características da rede de apoio (Granic \& Patterson, 2006; Pacheco e cols., 2005).

O modelo de Patterson foi construído em 1992 (Patterson e cols., 1992) e aprimorado no estudo em conjunto com Granic (Granic \& Patterson, 2006), no qual articularam as ideias anteriores à abordagem dos sistemas dinâmicos, propondo um modelo teórico para a compreensão dos problemas de comportamento. Nessa abordagem avançou-se no entendimento dos comportamentos antissociais, integrando as escalas temporais micro (momento a momento) e macrossociais (numa perspectiva desenvolvimentalista) para o desenvolvimento deste quadro. A abordagem também explica a etiologia do quadro através da inter-relação entre eventos e fatores contextuais, fatores biológicos, comportamentos parentais e infantis para a construção de padrões de interação hostis e agressivos, gerando os problemas de comportamento na infância e, longitudinalmente, podendo evoluir ao quadro Antissocial. O modelo descreve que as interações parentais coercivas, através de mecanismos de feedback positivo (reforçando-as) e negativos (mantendo-as) transformam-se em padrões de interação ou estados estáveis nas rela- 
ções pais-filhos. Tais padrões ou estados estáveis são chamados de "atratores", uma vez que possuem esta função na dinâmica familiar e, dessa forma, se tornam predizíveis na mesma.

Dentro desta perspectiva, os padrões coercivos não são entendidos como unidirecionais, mas sim associados a múltiplas e recíprocas causas. Assim, processos psicológicos (emoções e cognições) também são identificados como elementos importantes na manutenção de tais interações. No entendimento dos sistemas dinâmicos, as emoções costumam orientar a atenção dos indivíduos a elementos específicos de uma determinada situação. Assim, feedbacks positivos entre elementos emocionais e cognitivos se tornam a base das interpretações das situações, tanto nos momentos atuais como ao longo do tempo, desenvolvendo padrões de personalidade. Aponta-se que, as principais emoções, para pais e filhos envolvidos em padrões coercivos, são raiva e desprezo (Granic \& Patterson, 2006).

Desde o seu primeiro estudo (Patterson e cols., 1992) Patterson identificou que as condutas parentais, quando o quadro de problemas de comportamento está presente, tendem a mostrarem-se inconsistentes no reforçamento de comportamentos positivos, valorizando-os pouco e, ao mesmo tempo, fracassam na utilização de técnicas disciplinares que possam diminuir a frequência dos comportamentos desviantes. Desse modo, nas famílias onde se observa a presença de problemas de comportamento nos filhos tende a existir uma disciplina severa e inconsistente, assim como baixo envolvimento, monitoramento e supervisão parental (Pacheco e cols., 2005; Petit e cols., 1997; Reppold e cols., 2005).

Além da influência das práticas parentais, o modelo de Patterson e cols. (1992; Granic \& Patterson, 2006) traz importante contribuição ao destacar também as variáveis contextuais que favorecem o desenvolvimento de determinantes deste fenômeno. Estas variáveis contextuais apontadas pelo modelo são: nível socioeconômico (baixo), ser uma criança difícil (incluindo características como temperamento explosivo e recusa a aceitar críticas negativas), pais antissociais, altas taxas de criminalidade na vizinhança/comunidade (relação com iguais que apresentam desvios comportamentais), estressores (morte de um dos pais, divórcio, por exemplo) e avós (ou família de origem) com poucas habilidades sociais. Tais fatores contribuem para uma pobreza de habilidades de manejo familiar, principalmente quanto ao monitoramento, à disciplina, ao reforçamento positivo e à solução de problemas. Para tal modelo, o comportamento antissocial (termo utilizado no Modelo da Coerção) se desenvolve através de quatro estágios.

O primeiro estágio enfoca o "treinamento básico" (Patterson e cols., 1992), destacando que as variáveis contextuais só tendem a construir um comportamento antissocial e baixa autoestima na criança se associadas ao baixo monitoramento e supervisão parental; ou seja, nas técnicas educativas utilizadas pelos pais. A disciplina parental mostra-se, então, como principal variável no primeiro estágio descrito pelo modelo da coerção em face aos comportamentos infantis adequados ou inadequados. O segundo estágio é caracterizado pelas reações do meio frente ao problema de comportamento que a criança apresenta, evidenciando-se a rejeição parental e dos iguais, um baixo rendimento escolar e o humor deprimido. Frente aos princípios da abordagem dos sistemas dinâmicos, os processos de causalidade circular e feedbacks (positivo e negativo) explicam tais inter-relações (Granic \& Patterson, 2006). O terceiro estágio é qualificado em função dos fracassos acadêmicos e sociais, que parecem "empurrar" a criança/adolescente, que não desenvolveu habilidades sociais, a procurar pares com as mesmas características e comportamentos que ele. Assim, pode ocorrer a inserção do jovem num grupo desviante, o início do (ab)uso de substâncias e o comportamento delinquente. Por fim, o quarto estágio reflete os resultados de desajustamento de tais sujeitos, que são caracterizados por dificuldades em manter um emprego e uma relação afetiva, com a presença de crises constantes. Nesse estágio, o indivíduo pode ser institucionalizado por crimes ou desordem mental (Pacheco e cols., 2005; Patterson e cols., 1992).

O modelo da coerção (Granic \& Patterson, 2006; Patterson e cols., 1992) destaca a importância dos fatores contextuais, especialmente os parentais, na construção do comportamento antissocial. Esse modelo evidencia a importância das técnicas educativas empregadas pelos pais para disciplinar seus filhos e promover melhores níveis de saúde mental dos mesmos. Nesse sentido, a ampliação do modelo da coerção em face à abordagem dos sistemas dinâmicos, promove um aprofundamento no entendimento das interações parentais, explicados através da causalidade circular e dos feedbacks, fazendo como que estes se tornem padrões e perdurem no desenvolvimento infantil. Observar-se-á outros modelos que contemplam tal amplitude e destacam as relações parentais, diferenciando-se, porém, epistemologicamente, no entendimento das interações humanas. Sendo assim, a complexidade do tema permite que tomemos outras 
"lentes" para vislumbrarmos olhares distintos sobre o mesmo.

\section{Os problemas de comportamento à luz da psicanálise: Os estudos de Anna Freud}

Apesar da existência de um número grande de autores psicanalíticos que buscaram entender o comportamento infantil a partir dos processos inconscientes (e sua influência nos processos conscientes), alguns deles se destacaram, notadamente, no enfoque de comportamentos "normais" e "patológicos", com especial atenção ao que atualmente chamamos de problemas de comportamento. Para fins deste estudo, privilegiamos as ideias de Anna Freud, que contribuíram de forma expressiva com o estudo do desenvolvimento infantil.

Anna Freud (1976) parte da definição de normalidade na infância, buscando, num movimento contrário, distinguir e demarcar possíveis comportamentos patológicos no desenvolvimento do ego da criança. A partir deste referencial, buscaremos aprofundar a compreensão e a identificação de preditores dos problemas de comportamento a partir desta visão psicanalítica. A demarcação de problemas de comportamento como uma conduta patológica, assim como de diferentes quadros clínicos, é bastante complexa durante a infância. Entende-se que o equilíbrio mental do ser humano é baseado, em certa medida, em relações fixas entre as operações internas e também pela personalidade total e as condições ambientais oferecidas. Segundo A. Freud (1976), tais relações podem ser alteradas por aumento ou declínio dos impulsos do id, das forças do ego e do superego. No caminho da evolução da criança para a maturidade, entende-se que um conjunto de fatores se combina influenciando o seu funcionamento psíquico, podendo abalar, interromper, distorcer e desviar as forças que fundamentam o crescimento normal.

O mais importante para construir e explicar um diagnóstico é compreender a patogênese metapsicológica, visto que muitos comportamentos e sintomas são semelhantes a um mesmo quadro clínico. Ou seja, comportamentos como mentir e roubar, atitudes agressivas e destrutivas, atividades perversas, etc. presentes nos quadros de problemas de comportamento - não podem ser classificados como "normais ou patológicos' sem um olhar sobre as sequências de desenvolvimento individuais (Freud, 1976).

Assim, a partir desse entendimento psicanalítico, uma importante tarefa desenvolvimental da latência (que compreende as idades entre 7 e 11 anos) é a socia- lização das crianças. A latência é um período considerado fundamental na solidificação desta tarefa e da aprendizagem, através da sublimação. No entanto, tal processo tem início já nos primeiros anos de vida da criança, quando esta vai adquirindo certo domínio, responsabilidade e governo do seu corpo (Freud, A., 1976; Coll, Palacios \& Marchesi, 1995). A socialização, segundo Anna Freud (1976), depende da progressão do princípio do prazer para o princípio da realidade. Esse é associado ao desenvolvimento de determinadas funções do ego, como a fala, a introdução da razão e da lógica nos processos de pensamento, a compreensão de causa e efeito, e também o controle do ego sobre as ações e comportamentos impulsionados pelos desejos do id. Por isso, o avanço do princípio do prazer ao princípio da realidade implica na crescente tolerância à frustração e protelação de impulsos e desejos, assim como sua inibição e deslocamento. Porém, dentre estes fatores, a tolerância à frustração destaca-se como fator decisivo no processo de socialização, já que a sua ausência ou deficiência seria uma das principais razões do comportamento antissocial e delinquente.

O processo de socialização é complementado pelos mecanismos do ego de imitação, identificação e introjeção. A imitação de atitudes parentais é o mais primitivo destes mecanismos, e tem como função mostrar e fazer a criança experimentar o controle da satisfação de necessidades e impulsos. Já a identificação parte das imitações e da condição de que estas tenham tido resultados agradáveis. Através desta, as crianças buscam possuir permanentemente esses registros agradáveis, transportando os valores/ideias sociais dos pais e do mundo externo para o interno. Ao agregar estes valores ao seu ego, e se identificando com eles, a criança constrói os precursores do ego ideal e, consequentemente, do superego. Através da introjeção da autoridade parental (realizada durante e após o período edípico), o comportamento infantil se torna menos dependente dos pais, pois o controle vai deixando de ser externo (relacionado à figura dos mesmos) para se tornar interno (Duarte, 1989; Freud, A., 1976).

No quadro de problemas de comportamento o processo de identificação se dá de forma desintegrada, seja por meio de separações, rejeições ou outras interferências nos vínculos emocionais construídos entre os pais e a criança (Bowlby, 1969/1990). Os modelos de identificação parental na construção da identidade infantil influem no processo de socialização da criança, destacando as interações parentais e convergindo às ideias apontadas no "modelo de coerção", ainda 
que através de processos distintos. É através dessas relações objetais iniciais, entre a criança e seus cuidadores, que se dá a fusão das raízes libidinais e agressivas do id, importante função maturacional no ego infantil. Assim, na perspectiva psicanalítica, os problemas de comportamento ressaltam as dificuldades no relacionamento com os outros e com as leis externas, indicando a importância do papel educativo da família e da escola como favorecedoras do processo de socialização.

Um fator importante relacionado aos problemas de comportamento é a agressividade, assim como as alterações na sua expressão. A agressão em sua forma "pura" tende a ser não controlável, tanto externamente pelos pais quanto internamente pelo ego e superego, sendo necessário que novas vinculações objetais e o fortalecimento de processos libidinais atuem restabelecendo a fusão agressão-libido (Freud, A., 1976; Winnicott, 1965/1987). A agressão é compreendida como uma reação frente à frustração dos desejos e imposição do princípio da realidade, sendo que a falta desta imposição também pode provocar respostas no sentido de não ter a quem ou a que se opor. Por isso, destaca-se a importância das figuras educativas (pais e professores) imporem determinadas frustrações para que a agressão e a violência adquiram um valor simbólico e não só concreto. Um comportamento violento é comparado às "atuações" (acting-out), que são expressões primitivas dos impulsos, sem passar pela consciência, pelo pensamento. Essas expressões, na perspectiva psicanalítica, são entendidas como descargas, que devem adquirir na relação com o outro um sentido, um significado, para então sair do real e inserir-se no registro simbólico (Freller, 2001; Kupfer, 2000).

A partir desta perspectiva, assim como no modelo da coerção apresentado anteriormente, tanto a relação parental e/ou dos cuidadores como o ambiente são elementos fundamentais no processo de construção dos problemas de comportamento na infância. No entanto, o que é destacado a partir deste referencial teórico é que o registro simbólico/interno, tanto das relações como do ambiente, não são comuns a todos os sujeitos, não são objetivamente conhecidos nem suas repercussões são totalmente previsíveis. Por isso, parece-nos pertinente questionarmos sobre os fatores que compõem e interferem neste ambiente e nas relações parentais. Além disso, refletimos sobre a importância das relações familiares e não só parentais, sobre a reciprocidade entre o sujeito e a família. Portanto, ampliar a reflexão e análise do contexto e dos proces- sos familiares sobre as funções parentais e de outras contribuições na construção dos problemas de comportamento na infância pode possibilitar novos vértices para a compreensão deste fenômeno.

\section{Os problemas de comportamento à luz da teoria ecológico-sistêmica: 0 modelo de Belsky e os múltiplos olhares}

Um modo de pensar sistêmico caracteriza-se pela compreensão da conduta individual interdependente do contexto em que esta ocorre, uma vez que a epistemologia sistêmica entende que o que sabemos depende de como sabemos (Dowling, 1996; Nichols \& Schwartz, 1998; Vasconcellos, 2002). Um olhar sistêmico aos preditores dos problemas de comportamento na infância refere-se tanto à teoria familiar sistêmica como ao modelo ecológico (Belsky, 1981; Bronfenbrenner, 1996), visto que as relações entre a família e os demais sistemas, como a escola, são interdependentes no que se refere à educação de crianças. Um dos principais conceitos da teoria sistêmica, que é utilizado para explicar e compreender boa parte das interações sociais e familiares dos indivíduos é o da circularidade. Este conceito se refere às sequências de interação e padrões repetitivos que contribuem à perpetuação de um problema (Vasconcellos, 2002). Perguntamos: como ocorrem as interações familiares que contribuem para que uma criança continue a ser agressiva? A partir da circularidade, entendemos que o fato de os pais agirem de uma forma mais rígida com seu filho - por exemplo - por acreditarem que este terá reações violentas, tende a contribuir com um comportamento agressivo do filho, que reage às condutas rígidas e autoritárias (ou muito permissivas, por exemplo) de forma agressiva ou rebelde, justificando as crenças dos pais de que precisam ser rígidos com seu filho. Este conceito associa-se à reciprocidade presente nas interações humanas e, especialmente, nas interações pais e filhos, conforme a teoria sistêmica.

Destacamos outros dois conceitos extremamente importantes e inter-relacionados para a compreensão das interações e do funcionamento familiar: homeostase e feedback. O conceito de homeostase destaca que a família, como qualquer sistema/organismo vivo, possui uma tendência a buscar um estado permanente de equilíbrio (Dowling, 1996; Nichols \& Schwartz, 1998). A homeostase é o fenômeno através do qual a família busca manter seu status quo frente aos problemas e suas resistências quanto às mudanças. $\mathrm{Ou}$ como Hoffman (citado por Dowling, 1996) prefere defini-lo, a "coerência", destacando a forma como as 
"peças do sistema familiar" (p. 26) se encaixam, tanto interna quanto externamente.

Assim, o conceito de homeostase chama a atenção também para as propriedades autorreguladoras do sistema familiar, que poderão indicar o sentido/significado que os problemas representam neste. Igualmente, a homeostase só é possível a partir do uso que o sistema faz das informações que provém do ambiente externo na forma de feedbacks. Entendemos por circuito de feedback o intercâmbio de informações no qual o sentido das mesmas pode ser modificado por sua interação com os outros, tendo, novamente, um processo circular (Dowling, 1996). Tal conceito, que possui origens nas teorias comportamentais, distingue-se do referencial cognitivo-comportamental por incluir-se numa análise mais ampla, tendo em vista as interações dentro dos sistemas e não só dualmente. $\mathrm{O}$ processo de feedback ressalta a circularidade e a relação dinâmica que se estabelece entre os membros e o sistema familiar, assim como entre estes e outros sistemas, destacando a interdependência. Nesta sequência cíclica, pode-se observar que um mesmo comportamento pode ser tanto a causa como a consequência de um determinado padrão de inter-relação. Tal situação dependerá de onde se pontua a realidade, de onde parte o olhar do pesquisador/terapeuta (Dowling, 1996). Portanto, os problemas de comportamento podem ser entendidos à luz da teoria sistêmica como oriundos dos processos de feedback e circularidade. Ou seja, as sequências de interação familiar podem estar se dando de forma a reforçar o problema de comportamento da criança, assim como mecanismos de feedback podem estar atuando para a manutenção deste padrão. Dessa forma, os estudos empíricos que ressaltam as práticas educativas como possíveis preditoras dos problemas de comportamento, certamente vêm ao encontro deste olhar sistêmico, buscando aproximar-se do mesmo.

Um modelo amplamente utilizado por autores que se fundamentam no referencial ecológico-sistêmico para explicar e compreender os problemas de comportamento na infância é o de Belsky (1981, 1984, 1990; Belsky, Steinberg, \& Draper, 1991). Este modelo, que é construído a partir do marco ecológico, tem sido presença constante nos periódicos científicos e se destaca por agregar à compreensão dos problemas de comportamento na infância e adolescência tanto os aspectos individuais da criança e de seus pais, como relacionais e contextuais do sistema familiar. No seu modelo, Belsky $(1981 ; 1984)$ destaca três instâncias fundamentais de uma parentalidade eficiente: as ca- racterísticas dos pais, as características das crianças/adolescentes e as características do contexto social onde o exercício da parentalidade acontece. Explicando especificamente o contexto social do exercício da parentalidade, Belsky (1984) afirma que dele fazem parte a rede de apoio social (como a escola e os amigos, por exemplo), a relação conjugal e as experiências ocupacionais dos pais. Esse modelo assume também que a história desenvolvimental dos pais e as características temperamentais da criança, assim como o relacionamento conjugal, a rede de apoio social e o trabalho dos pais influenciam a personalidade e o bem-estar geral dos mesmos. Consequentemente, a funcionalidade da parentalidade e do desenvolvimento infantil serão transformados a partir de tais interferências.

Compreende-se então, que os problemas de comportamento na infância e a parentalidade são fenômenos associados, interdependentes e multideterminados. A parentalidade mostra-se multideterminada, já que as características individuais dos pais e da criança e a rede de apoio social possuem valores explicativos diferentes em cada relação e contexto, podendo mostrar-se como contribuidoras ou dificultadoras da promoção de uma parentalidade funcional (Belsky, 1984). Além disso, o modelo teórico de Belsky (1981, 1984, 1990; Belsky e cols., 1991) destaca a importância dos diferentes padrões de interação entre pais e filhos e seus efeitos sobre o desenvolvimento uns dos outros. Estes padrões podem se construir através das variantes entre os três elementos da parentalidade; como, por exemplo, numa família pode ocorrer que pais mais autoritários, tenham filhos mais agressivos, rede de apoio social pouco eficaz e problemas conjugais (Belsky e cols., 2001). A família, como um sistema de interação e interdependência, mantém relações com outros sistemas, tais como a escola, a vizinhança, o trabalho dos adultos, os serviços de saúde, entre outros. Essa ideia de interdependência foi descrita por Bronfenbrenner (1996), quando apresentou o modelo ecológico-contextual. A compreensão da interdependência do contexto, em suas distintas dimensões (família, comunidade, ideologias) e o indivíduo, é o ponto comum entre o modelo teórico de Belsky e Bronfrenbenner. Nesse sentido, ambos os modelos que tem se mostrado bastante importantes para analisar a interação família-escola frente aos problemas de comportamento de crianças.

Estudos empíricos nacionais e internacionais têm apontado a importância de distintos aspectos do micro e do mesosistema como influentes na ocorrência de problemas de comportamento na infância. Num estu- 
do paulista (Ferreira \& Marturano, 2002), realizado com 114 crianças de grupos clínicos e não clínicos, identificou-se aspectos do relacionamento parental, da rede de apoio social e de recursos materiais, associados a fatores de adversidade familiar, que contribuíram para o desenvolvimento de problemas de comportamento na idade escolar. As práticas educativas parentais coercitivas, como o uso de ameaças, neste estudo mostraram-se inadequadas e ineficazes, dificultando as interações sociais das crianças. Por conta disso, observou-se também que a disponibilidade de materiais apoiadores do desenvolvimento infantil (associada aos recursos financeiros da família) foi facilitadora para o aproveitamento escolar das mesmas e que a existência de uma rede de apoio aos pais mostrou-se um elemento incrementador de práticas educativas mais eficazes frente aos problemas de comportamento. Os resultados deste estudo mostram que o desenvolvimento dos problemas de comportamento na infância está associado às interações parentais num contexto onde as adversidades parecem afetar o funcionamento familiar, ideia reiterada por Olson e cols. (2000).

Corroborando estas ideias, os resultados de um estudo americano (Dodge e cols., 1994), utilizando uma grande amostra (585 crianças e suas mães), também evidenciaram que aspectos contextuais, como nível socioeconômico desfavorecido, conviver em comunidades com alto índice de violência, presença frequente de eventos estressores na família e pouco apoio social para a mãe, foram aspectos influentes para o desenvolvimento de problemas de comportamento. Nesse sentido, as relações do mesosistema mostraram-se determinantes, pois os autores entenderam que o fato de viver em comunidades violentas pode fazer com que os pais tenham medo de que seus filhos estejam em risco de serem vitimizados ou vitimizarem outros. Assim, podem ser particularmente sensíveis ao mau comportamento de seus filhos e responder a estes sinais através de formas extremamente coercitivas de controle externo. Dentro dessa perspectiva, este padrão parental pode ter efeitos paradoxais na promoção de comportamentos antissociais, pois se entende que certo grau de agressividade na criança pode tornar-se adaptativo em comunidades com índices elevados de violência. Sendo assim, os pais acabam reforçando a agressividade como um desejo positivo de socializar seus filhos de forma defensiva (Petit e cols., 1997). Esses resultados reforçam a importância do conhecimento do contexto para a avaliação de um comportamento e a identificação de onde parte o olhar daquele que avalia o fenômeno, destacando a importância do modelo ecológico-sistêmico.

Corroborando o modelo de Belsky (1981), alguns estudos (Belsky e cols., 2001; Bolsoni-Silva \& Marturano, 2002; Hart, Ladd \& Burleson, 1990; Jouriles e cols., 1991) também destacaram a importância do relacionamento conjugal na eficácia das práticas educativas parentais. Conforme estes estudos, tanto a baixa satisfação conjugal como o desacordo na função educativa, contribuem para a incoerência e o estresse parental que se reflete em problemas de comportamento das crianças, especialmente em meninos (Belsky, 1981; Jouriles e cols., 1991). Assim, reiterase a interdependência dos subsistemas familiares e dos sistemas em geral frente à existência de problemas de comportamento infantil. É importante então, atentar para as relações estabelecidas no contexto socializador da criança, tanto na família como nos sistemas com os quais esta se relaciona. Conforme Dowling (1996), uma visão sistêmica da realidade considera que a conduta dos indivíduos (das famílias ou dos sistemas) está intimamente relacionada ao contexto onde se produzem.

Destaca-se o modelo de Belsky como preventivo, além de integrador, pois as dimensões de estudo propostas por ele (pais/crianças/contexto social) podem ser entendidas tanto como fatores de risco, como podem tornar-se fatores de proteção no desenvolvimento dos problemas de comportamento. Sobretudo, utilizar conhecimentos que visem ampliar a compreensão de fenômenos, tais como os problemas de comportamento, devem contribuir para a construção de propostas de reflexão e intervenção frente à perpetuação de ações que reproduzem a violência e, assim, promover comportamentos pró-sociais. Igualmente, a possibilidade de "aproximar" e "afastar" o foco sobre o fenômeno em estudo, possibilita que se mantenha a atenção tanto aos aspectos intra como extrafamiliares. Tal razão sustenta a escolha de um olhar integrativo, que não privilegie um elemento em detrimento de outros. Por vezes, pode mostrar-se desafiadora uma postura que vise compreender tantos elementos frente a um fenômeno a ser investigado, mas acreditamos que essa possibilidade mostra-se tão complexa como nosso objeto de estudo.

\section{CONSIDERAÇÕES FINAIS}

A partir das ideias revisadas até então, torna-se evidente a convergência entre os modelos teóricos apresentados a respeito da importância das relações parentais e do contexto familiar no desenvolvimento de crianças e adolescentes. Mais especificamente, 
destaca-se o papel do ambiente, das relações parentais e da posição que o indivíduo se encontra nessa interrelação como preditores dos problemas de comportamento. Nesse sentido, as práticas educativas parentais poderão ou não se tornar fatores promotores da socialização infantil. De forma geral, muitos argumentos mostram-se comuns, mas diferenciam-se, como os grandes campos epistêmicos, por seu foco e sua visão de homem, especialmente dos determinantes da personalidade humana.

A socialização é tarefa primordial na família, objetivando fornecer recursos e habilidades psicológicas para as crianças relacionarem-se com o outro, assim como, apontar e orientar os comportamentos dos filhos. Tal tarefa possui implicações nas relações da família com outros sistemas e, até mesmo, dentro dela. Como foi descrito, é uma tarefa complexa, que exige não só informações, coerência e recursos psíquicos dos pais, como do ambiente no qual a família está inserida. Nesse sentido, o fracasso nessa tarefa pode ocasionar problemas crônicos e de grande repercussão no desenvolvimento dos seus membros.

Muitos aspectos da sociedade atual (como a violência, os preconceitos e o hedonismo) têm se mostrado entraves ao desenvolvimento deste processo de socialização. Por isso, pensamos que é necessário um exame cuidadoso dos distintos elementos dos contextos de socialização das crianças, para que haja uma compreensão abrangente ao ponto de explicar como esse processo se dá na contemporaneidade. Preocuparse com rendimento escolar, ocorrência ou não de eventos estressores na família e a intensidade/proximidade da violência, parece-nos inevitável atualmente. Acreditamos que o cuidado parental deve fornecer recursos e habilidades psicológicas através da socialização primária (intrafamiliar) e do fortalecimento da relação pais-filhos para que as crianças possam enfrentar de forma menos "despreparada" a tarefa de socialização no ambiente extrafamiliar.

No núcleo das relações familiares, como destacado anteriormente, as interações parentais seriam unidades de análise deste processo, interdependentes ao funcionamento familiar e à comunidade onde se inserem. Nos modelos teóricos apresentados este foi elemento consensual, tanto preditivo como preventivo, para o desenvolvimento dos problemas de comportamento. Destaca-se a convergência, especificamente, entre os modelos da coerção e ecológico-sistêmico, inclusive na utilização de conceitos, para a compreensão das interações familiares e contextuais, o que reitera a relevância de ambos. Da mesma forma, ao identificar- se processos semelhantes percebe-se que, mesmo sob o olhar de 'lentes' distintas, as interações parentais e sua funcionalidade ou disfuncionalidade podem ser mantidas no funcionamento familiar reverberando sobre a qualidade das demais relações e do desenvolvimento psicológico de todos seus membros.

Assumimos, portanto, face às considerações e reflexões, um olhar ecológico-sistêmico frente aos problemas de comportamento, já que este exige a consideração de todos os elementos como causas e como consequências simultaneamente, valorizando também a relação com outros sistemas (como a escola, por exemplo). Entretanto, esses elementos devem ser analisados com "pesos" diferentes, determinados conforme as especificidades de um marco teórico, que postula um entendimento do homem e suas relações. No entanto, ao focar-se na interação de pais e filhos, muitas vezes, pode vir em detrimento das idiossincrasias do(s) indivíduo(s). Nesse sentido, os aspectos de tal ordem podem ficar difusos a partir desta lente teórica, ocultando fenômenos mais específicos e particulares do indivíduo ou caso em estudo. Nesse sentido, as contribuições do modelo psicanalítico atentam para estas lacunas, relativizando muitos postulados e tornando mais complexo e particular o entendimento do fenômeno.

Acredita-se ser necessário, igualmente, atentar sobre as relações entre o meio ambiente e as condições que ele oferece ao desenvolvimento de uma parentalidade eficiente. Ou ainda, sobre a possibilidade do sistema parental reverberar tais condições oferecidas (ou a falta delas), tanto de forma positiva (como fatores protetivos) como negativa (como fatores de risco) no seu desenvolvimento. Por isso, é necessário que examinemos também as relações estabelecidas entre os sistemas educativos da infância, para compreender a forma de interação dos mesmos e auxiliar na potencialização de suas ações. Acreditamos que assim, podemos identificar pontos de congruência e incongruência, convergência e divergência, continuidade e descontinuidade nas inter-relações entre os sistemas envolvidos nesse processo.

Assim, concluímos como necessária a ampliação do olhar restrito à família, incluindo novos sistemas que fazem parte desta interação educativa. Por isso, a escola, como contexto igualmente educativo, se mostra um espaço importante para o aprofundamento do processo de socialização, em relação aos sucessos e fracassos da criança e dos sistemas (família e escola) envolvidos nessa tarefa. Novos estudos devem voltar o seu olhar para essas relações e às problemáticas daí decorrentes. 


\section{REFERÊNCIAS}

Achenbach, T. M., \& Howell, C. T. (1993). Are american children's problems getting worse? A 13-year comparison. Journal of American Academy on Child and Adolescent Psychiatry, 32, 1145-1154.

Achenbach, T. M. (1991). Manual for the Child Behavior Checklist / 4-18 and 1991 profile. Burlington, VT: University of Vermont, Department of Psychiatry.

Alvarenga, P., \& Piccinini, C. (2001). Práticas educativas maternas e problemas de comportamento em pré-escolares. Psicologia: Reflexão e Crítica, 14, 449-460.

Baumrind, D. (1965). Parental control and parental love. Children, $12,230-234$.

Baumrind, D. (1996). Parenting: The discipline controversy revisited. Family Relations, 15, 405-414.

Belsky, J. (1981). Early human experience: A family perspective. Developmental Psychology, 17, 3-23.

Belsky, J. (1984). The determinants of parenting: A process model. Child Development, 55, 83-96.

Belsky, J. (1990). Parental and nonparental child care and children's socioemotional development: A decade in review. Journal of Marriage and the Family, 52, 885-903.

Belsky, J., Jaffee, S., Hsieh, K., \& Silva, P. A. (2001). Childrearing antecedents of intergenerational relations in young adulthood: A prospective study. Developmental Psychology, 37, 801-813.

Belsky, J., Steinberg, L., \& Draper, P. (1991). Childhood experience, interpersonal development and reproductive strategy: An evolutionary theory of socialization. Child Development, 62, 647-670.

Bolsoni-Silva, A. T., \& Marturano, E. M. (2002). Práticas educativas e problemas de comportamento: Uma análise à luz das habilidades sociais. Estudos de Psicologia, 7, 227-235.

Bordin, I. A. S., Mari, J. J., \& Caeiro, M. F. (1995). Validação da versão brasileira do "Child Behavior Checklist" (CBCL) (Inventário de Comportamentos da Infância e Adolescência): Dados preliminares. Revista ABP-APAL, 17, 55-66.

Bowlby, J. (1990). Apego: Vol. 1 (A. Cabral, Trad.). São Paulo: Martins Fontes. (Original publicado em 1969)

Bronfenbrenner, U. (1996). A ecologia do desenvolvimento humano: Experimentos naturais e planejados (M. A. Verissimo, Trad.). Porto Alegre: Artes Médicas. (Original publicado em 1979)

Ceballos, E., \& Rodrigo, M. J. (2003). Las metas y estrategias de socialización entre padres e hijos. Em M. J. Rodrigo \& J. Palacios (Orgs.), Familia y desarrollo humano (pp. 225-244). Madrid: Alianza Editorial.

Coll, C., Palacios, J., \& Marchesi, A. (1995). Desenvolvimento psicológico e educação: Psicologia evolutiva. Porto Alegre: Artes Médicas.

Darling, N., \& Steinberg, L. (1993). Patenting style: An integrative model. Psychological Bulletin, 113, 487-496.

Deslandes, R., Bouchard, P., \& St-Amant, J. (1998). Family variables as predictors of school achievement: Sex differences in Quebec adolescents. Canadian Journal of Education, 23, 390404.

Dodge, K. A., Petit, G. S., \& Bates, J. E. (1994). Socialization mediators of the relation between socioeconomic status and child conduct problems. Child Development, 65, 649-665.
Dowling, E. (1996). Marco teórico: Un enfoque sistémico conjunto de los problemas educativos con niños. Em E. Dowling \& E. Osborne, Familia y escuela: Una aproximación conjunta y sistémica a los problemas infantiles (pp. 21-50). Buenos Aires: Paidós.

Duarte, I. (1989). O tratamento de crianças com transtornos de conduta. Em I. Duarte, I. Bornholdt, \& M. G. K. Castro (Orgs.), A prática da psicoterapia infantil (pp. 131-145). Porto Alegre: Artes Médicas.

Ferreira, F. C., \& Marturano, E. M. (2002). Ambiente familiar e os problemas de comportamento apresentados por crianças com baixo desempenho escolar. Psicologia: Reflexão e Crítica, 15, 35-44.

Freller, C. C. (2001). Histórias de indisciplina escolar: O trabalho de um psicólogo numa perspectiva winnicottiana. São Paulo: Casa do Psicólogo.

Freud, A. (1976). Infância normal e patológica: Determinantes do desenvolvimento (A. Cabral, Trad.). Rio de Janeiro: Zahar. (Original publicado em 1965)

Glaskow, K. L., Dornbusch, S. M., Troyer, L., Steinberg, L., \& Ritter, P. L. (1997). Parenting styles, adolescents'attributions, and educational outcomes in nine heterogenoeus high schools. Child Development, 68, 507-529.

Granic, I., \& Patterson, G. R. (2006). Toward a comprehensive model of antisocial development: A dynamic systems approach. Psychological Review, 113, 101-131

Grusec, J. E., \& Goodnow, J. J. (1994). Impact of parental discipline methods on the child's internalization of values: A reconceptualization of current points of view. Developmental Psychology, 30, 04-19.

Hart, C. H., Ladd, G. W., \& Burleson, B. R. (1990). Children's expectations of the outcomes of social strategies: Relations with sociometric status and maternal disciplinary styles. Child Development, 61, 127-137.

Hoffman, M. L. (1975). Moral internalization, parental power, and the nature of parent-child interaction. Developmental Psychology, 11, 228-239.

Hoffman, M. L. (1994). Discipline and internalization. Developmental Psychology, 30, 26-28.

Jouriles, E. N., Murphy, C. M., Farris, A. M., Smith, D. A., Richters, J. E., \& Waters, E. (1991). Marital adjustment, parental disagreements about child rearing, and behavioral

problems in boys: Increasing the specificity of the marital assessment. Child Development, 62, 1424-1433.

Kupfer, M. C. M. (2000). Educação para o futuro: Psicanálise e educação. São Paulo: Escuta.

Nichols, M., \& Schwartz, R. (1998). Terapia familiar: Conceitos e métodos (M. F. Lopes, Trad.). Porto Alegre: Artes Médicas.

Olson, S. L., Bates, J. E., Sandy, J. M., \& Lanthier, R. (2000). Early developmental precursors of externalizing behavior in middle childhood and adolescence. Journal of Abnormal Child Psychology, 28, 119-133.

Pacheco, J. (2002). A construção do comportamento antissocial em adolescentes autores de atos infracionais: Uma análise a partir das práticas educativas e dos estilos parentais. Projeto de tese de doutorado não publicado, Universidade Federal do Rio Grande do Sul, Porto Alegre.

Pacheco, J., Alvarenga, P., Reppold, C., Piccinini, C. A., \& Hutz, C. S. (2005). Estabilidade do comportamento antissocial na transição da infância para a adolescência: Uma perspectiva desenvolvimentista. Psicologia: Reflexão e Crítica, 18, 55-61. 
Patterson, G., Reid, J., \& Dishion, T. (1992). Antisocial boys. Eugene, OR: Castalia.

Pettit, G. S., Bates, J. E., \& Dodge, K. A. (1997). Supportive parenting, ecological context, and children's adjustment: A seven-year longitudinal study. Child Development, 68, 908923.

Reppold, C. T., Pacheco, J., Bardagi, M., \& Hutz, C. S. (2002). A importância da interação familiar para a prevenção de problemas de comportamento e o desenvolvimento de competências psicossociais em crianças e adolescentes: Uma análise das práticas educativas e dos estilos parentais. Em C. S. Hutz (Org.), Situações de risco e vulnerabilidade na infância e na adolescência: Aspectos teóricos e estratégias de intervenção (pp. 0751). São Paulo: Casa do Psicólogo.

Reppold, C. T., Pacheco, J., \& Hutz, C. (2005). Comportamento agressivo e práticas disciplinares parentais. Em C. S. Hutz (Org.), Violência e risco na infância e adolescência: Pesquisa e intervenção (pp. 9-42). São Paulo: Casa do Psicólogo.

Rodrigo, M. J., Janssens, J. M. A. M., \& Ceballos, E. (1999). Do children's perceptions and attributions mediate the effects of mothers'child-rearing actions? Journal of Family Psychology, $13,508-522$.
Shelton, K. K., Frick, P. J., \& Wooton, J. (1996). Assessment of parenting practices in families of elementary school-age children. Journal of Clinical Child Psychology, 25, 317-329.

Vasconcellos, M. J. E. (2002). Pensamento sistêmico: O novo paradigma da ciência. Campinas: Papirus.

Winnicott, D. W. (1987). Privação e delinquência (M. B. Cipolla, Trad.). São Paulo: Martins Fontes. (Original publicado em 1965)

Zdanowicz, N., Pascal, J., \& Reynaert, C. (2004). ¿Juega la familia un papel clave en la salud durante la adolescencia?. The European Journal of Psychiatry, 18, 215-224.
Recebido: $24 / 08 / 2008$ Última revisão: 18/07/2011 Aceito: $30 / 08 / 2011$ 